\title{
Roles of the Insular Cortex in the Modulation of Pain: Insights from Brain Lesions
}

\author{
Christopher J. Starr, ${ }^{1}$ Lumy Sawaki, ${ }^{2}$ George F. Wittenberg, ${ }^{4}$ Jonathan H. Burdette, ${ }^{3}$ Yoshitetsu Oshiro, ${ }^{1}$ \\ Alexandre S. Quevedo, ${ }^{1}$ and Robert C. Coghill ${ }^{1}$ \\ Departments of ${ }^{1}$ Neurobiology and Anatomy, ${ }^{2}$ Neurology, and ${ }^{3}$ Radiology, Wake Forest University School of Medicine, Winston-Salem, North Carolina \\ 27157-1010, and ${ }^{4}$ Department of Neurology, University of Maryland School of Medicine, Baltimore, Maryland 21201-1544
}

Subjective sensory experiences are constructed by the integration of afferent sensory information with information about the uniquely personal internal cognitive state. The insular cortex is anatomically positioned to serve as one potential interface between afferent processing mechanisms and more cognitively oriented modulatory systems. However, the role of the insular cortex in such modulatory processes remains poorly understood. Two individuals with extensive lesions to the insula were examined to better understand the contribution of this brain region to the generation of subjective sensory experiences. Despite substantial differences in the extent of the damage to the insular cortex, three findings were common to both individuals. First, both subjects had substantially higher pain intensity ratings of acute experimental noxious stimuli than age-matched control subjects. Second, when pain-related activation of the primary somatosensory cortex was examined during left- and right-sided stimulation, both individuals exhibited dramatically elevated activity of the primary somatosensory cortex ipsilateral to the lesioned insula in relation to healthy control subjects. Finally, both individuals retained the ability to evaluate pain despite substantial insular damage and no evidence of detectible insular activity. Together, these results indicate that the insula may be importantly involved in tuning cortical regions to appropriately use previous cognitive information during afferent processing. Finally, these data suggest that a subjectively available experience of pain can be instantiated by brain mechanisms that do not require the insular cortex.

\section{Introduction}

The insular cortex is often bilaterally activated during noxious somatosensory stimulation and has been suggested to play an important role in pain processing (Coghill et al., 1994, 1999). Afferent nociceptive information can be transmitted rostrally from the second somatosensory cortex (SII) to the posterior insula and then to the anterior insula. The reciprocal connections of the insula with the prefrontal cortex, anterior cingulate cortex (ACC), amygdala, parahippocampal gyrus, and SII can allow afferent nociceptive information to be integrated with information related to working memory, affect, and attention (Mufson et al., 1981; Mesulam and Mufson, 1982; Mufson and Mesulam, 1982; Friedman and Murray, 1986; Friedman et al., 1986).

Activation of the insular cortex has been correlated with the intensity of noxious stimulation, suggesting that this structure may play a role in pain intensity coding (Derbyshire et al., 1997; Coghill et al., 1999). Consistent with this role, psychophysical data indicate that patients with lesions affecting the posterior insula and parietal operculum, but not the anterior insula, exhibit elevated heat pain thresholds (Greenspan and Winfield, 1992;

\footnotetext{
Received 0ct. 27, 2008; revised Dec. 3, 2008; accepted Dec. 8, 2008.

This work was supported by National Institutes of Health Grant R01 NS39426. We thank the Functional Magnetic Resonance Imaging of the Brain Image Analysis Group, Oxford University, for the FSL analysis software, and Stephania Jordan for her help in collecting data for the experiment.

Correspondence should be addressed to Dr. Robert C. Coghill, Department of Neurobiology and Anatomy, Wake

Forest University School of Medicine, Winston-Salem, NC 27157-1010. E-mail: rcoghill@wfubmc.edu. D0I:10.1523/JNEUROSCI.5173-08.2009

Copyright $\odot 2009$ Society for Neuroscience $\quad$ 0270-6474/09/292684-11\$15.00/0
}

Greenspan et al., 1999). However, when tested with suprathreshold noxious stimuli, patients with insular lesions exhibit more complex alterations in their experience of pain. They exhibit pain asymbolia, a condition in which individuals can recognize noxious stimuli as painful but exhibit inappropriate affective responses and have difficulty in appraising the meaning and significance of such stimuli (Berthier et al., 1988).

The extensive connectivity of the insula suggests that it may play a complex, multifaceted role in pain. For example, activation of the insula during placebo, opioid analgesia, expectation, and hypnosis suggests that it may be important in both antinociceptive as well as pronociceptive processes (Petrovic et al., 2002; Lorenz et al., 2003; Derbyshire et al., 2004; Koyama et al., 2005; Zubieta et al., 2005; Kong et al., 2006, 2007; Craggs et al., 2007). Activity in the anterior insula can also modulate activation of the prefrontal cortex and anterior cingulate cortex in a task- or situation-dependent manner (Craggs et al., 2007; Sridharan et al., 2008). These findings suggest that the insula may be well positioned to use cognitive information to modulate connected brain areas involved in processing of sensory-discriminative, affective, and cognitive-evaluative components of pain. Accordingly, lesions of the insula would be expected to lead to a complex pattern of altered experiences of pain evoked by suprathreshold noxious stimuli. Such changes could even include increases in pain sensitivity because of a loss of modulation of brain areas involved in various aspects of nociceptive processing.

To date, few studies have investigated the integrity of pain perception and alteration in pain-related brain activation after 
damage to the insula. To investigate the role that the insula plays in pain processing and modulation, sensory testing and functional magnetic resonance imaging (fMRI) experiments were used to examine two patients who suffered from large left middle cerebral artery (MCA) ischemic strokes with lesions encompassing the insular cortex.

\section{Materials and Methods \\ Subjects \\ Two male stroke patients (age, 53 and 59) were recruited to participate in the study. Both patients suffered from large left MCA strokes with lesions encompassing the insular cortex (see Fig. 1, Table 1). A group of 14 healthy older adults was recruited to assess differences in pain percep- tion, sensory thresholds, and pattern of brain activations in lesioned subjects. There were eight females and six males (age, $46-75$ years; mean, 59 years). Of the 14 subjects, 13 participated in the imaging session. One female participant did not participate in the brain imaging session be- cause of claustrophobia. They underwent the same experimental proto- cols as the stroke patients. All study participants gave written, informed consent acknowledging that (1) they would experience experimental painful stimuli, (2) all methods and procedures were clearly explained, and (3) they were free to withdraw from the experiment at any time without prejudice. All of the procedures were approved by the Institu- tional Review Board of Wake Forest University School of Medicine.}

\section{Psychophysical data collection}

During the psychophysical training session, subjects practiced rating 32 noxious heat stimuli $\left(35,43-49^{\circ} \mathrm{C}, 5 \mathrm{~s}\right.$ duration) using a visual analog scale (VAS). The VAS is a $15 \mathrm{~cm}$ plastic sliding scale device widely used to assess pain because of ease of use while providing quantifiable measurements of pain intensity and pain unpleasantness [Parisian Novelty (Price et al., 1994)]. The minimum was anchored with "No pain sensation" or "Not at all unpleasant," whereas the maximum was anchored with "Most intense pain imaginable" or "Most unpleasant imaginable." Using an audio analogy, subjects were instructed to distinguish between pain intensity and pain unpleasantness (Price et al., 1989). The stimuli were applied to their dorsal calves via a $16 \times 16 \mathrm{~mm}^{2}$ Peltier device (Medoc TSA II) secured with a Velcro strap. Baseline temperature was maintained at $35^{\circ} \mathrm{C}$, and stimulus temperatures were delivered with rise and fall rates of $6^{\circ} \mathrm{C} / \mathrm{s}$ and were feedback controlled. These data are not reported further.

Next, subjects provided poststimulus pain intensity and pain unpleasantness VAS ratings of 18 graded noxious heat stimuli of three different temperatures $\left(35,45\right.$, or $\left.50^{\circ} \mathrm{C}\right)$ delivered at $5 \mathrm{~s}$ duration in a pseudorandom manner on each calf. To minimize sensitization, habituation, or hyperalgesia, all trials were separated by a minimum of $30 \mathrm{~s}$ and were performed on previously unstimulated sites of the skin (Pedersen and Kehlet, 1998a,b).

Finally, subjects received identical stimulus paradigms that would be used during the fMRI session to familiarize them with the temporal sequence of stimuli within a series to minimize variations in cognitive components such as expectation and anxiety.

\section{Quantitative testing of sensory thresholds}

Tactile thresholds. To quantitatively assess tactile thresholds and deficits of areas that may be affected by the lesions, von Frey filaments were used to examine the ventral forearms and dorsal calves bilaterally using the methods of constant stimuli. Minimum force (in newtons) required for subjects to consistently (greater than $\sim 75 \%$ ) detect touch for each of the areas was recorded. Each body area was tested a variable number of times as the threshold was successively approximated with different von Frey filaments.

Thermal thresholds. Heat pain threshold, cold pain threshold, warm detection threshold, and innocuous cool detection threshold of both right and left calves were determined by the method of limits. For each of the four modalities of interest, the $32 \times 32 \mathrm{~mm}^{2}$ thermode was applied to the dorsal calf. For warm detection and heat pain thresholds, the temperature was increased at $1^{\circ} \mathrm{C} / \mathrm{s}$ from 35 to $50^{\circ} \mathrm{C}$. Subjects were then asked to indicate either the transition point at which the baseline temperature transitions into a warm sensation (warm detection) or when nonpainful warm sensation changed into a painful heat sensation (heat pain) by pressing a button. For innocuous cool and cold pain thresholds, temperature was decreased at $1^{\circ} \mathrm{C} / \mathrm{s}$ from 35 to $0^{\circ} \mathrm{C}$. Subjects were subsequently asked to indicate the transition point at which the baseline temperature changed into a cool sensation (innocuous cool) or when nonpainful cool sensation transitions into a painful cold sensation (cold pain). For each of the modalities measured, the test was repeated successively six times and the mean threshold temperature was calculated. To minimize sensitization, habituation, or hyperalgesia, all trials were separated by a minimum of $30 \mathrm{~s}$ and were performed on previously unstimulated skin sites (Pedersen and Kehlet, 1998a,b).

\section{Functional imaging}

The fMRI session consisted of eight series (four during stimulation of the unaffected side, four during stimulation of the affected side, alternating between sides). Long duration noxious stimuli were delivered using a block design $\left(49^{\circ} \mathrm{C} ; 30 \mathrm{~s}\right.$ on $30 \mathrm{~s}$ off; five cycles) with baseline temperature of $35^{\circ} \mathrm{C}$. At the end of each fMRI series, the subjects were asked to provide overall pain intensity and unpleasantness VAS ratings. The dorsal calves were selected as sites of stimulation because the primary somatosensory cortex (SI) leg representations were far removed from the areas affected by the strokes. This ensures that results seen are attributable to lesions affecting the insular cortex, and not SI.

\section{Image acquisition and image processing}

fMRI data were acquired on a $1.5 \mathrm{~T}$ General Electric Twin-Speed LX Scanner with a birdcage quadrature head coil (General Electric Medical Systems). For functional imaging, blood oxygenation level-dependent images of the entire brain were acquired continuously by using singleshot echoplanar imaging [echo time (TE), $40 \mathrm{~ms}$; repetition time (TR), $2 \mathrm{~s} ; 28 \times 5$-mm-thick slices; in-plane resolution, $3.72 \times 3.75 \mathrm{~mm}$; flip angle, $90^{\circ}$; no slice gap] (Ogawa et al., 1990). Each fMRI series consisted of 165 volumes and lasted $350 \mathrm{~s}$ long with $20 \mathrm{~s}$ equilibration time at the beginning of each series. During each fMRI acquisition series, subjects were requested to close their eyes. High-resolution structural scans were acquired using a three-dimensional spoiled gradient-echo (threedimensional inversion spoiled gradient-recalled acquisition in a steady state) sequence (inversion time, $600 \mathrm{~ms}$; TR, $9.1 \mathrm{~ms}$; flip angle, 20 ${ }^{\circ}$; TE, $1.98 \mathrm{~ms}$; matrix, $256 \times 196$; section thickness, $1.5 \mathrm{~mm}$ with no gap between sections; 124 sections; in-plane resolution, $0.9375 \times 0.9375$ $\mathrm{mm}$; field of view, $24 \mathrm{~cm}$ ).

The functional image analysis package FSL [Functional Magnetic Resonance Imaging of the Brain (FMRIB) Software Library (Center for FMRIB, University of Oxford, Oxford, UK)] was used for image processing and statistical analysis. The functional data were movement corrected, spatially smoothed by $5 \mathrm{~mm}$ full-width at half-maximum with a threedimensional isotropic Gaussian kernel, and temporally filtered by a nonlinear high-pass filter with a cutoff period of $90 \mathrm{~s}$. Each functional image was scaled by its mean global intensity (intensity normalization). Next, each subject's functional images were registered to their structural data using a seven-parameter linear three-dimensional transformation and transformed into standard stereotaxic space (as defined by the Montreal Neurological Institute) using a 12-parameter linear three-dimensional transformation (Tailarach and Tournoux, 1988; Jenkinson et al., 2002). Using animated time series, visual inspection confirmed that spatial transformations during registration and movement correction were successfully accomplished.

\section{Statistical analysis of psychophysical data}

To examine the effects of insular lesions on pain, we compared pain intensity and unpleasantness ratings between affected and unaffected sides within each patient using both one-factor and two-factor ANOVAs (JMP software; SAS Institute). To determine whether lesioned subjects still retained the capacity to evaluate pain intensity evoked by graded noxious stimuli $\left(35,45,50^{\circ} \mathrm{C} ; 5 \mathrm{~s}\right)$, two-factor within-subjects ANOVAs were used to examine the effects of body side and stimulus temperature on pain intensity and unpleasantness ratings within each patient. For long duration noxious stimulation during fMRI $\left(49^{\circ} \mathrm{C} ; 30 \mathrm{~s}\right.$ on $30 \mathrm{~s}$ off; five cycles), we used separate ANOVAs (affected and unaffected sides) to 


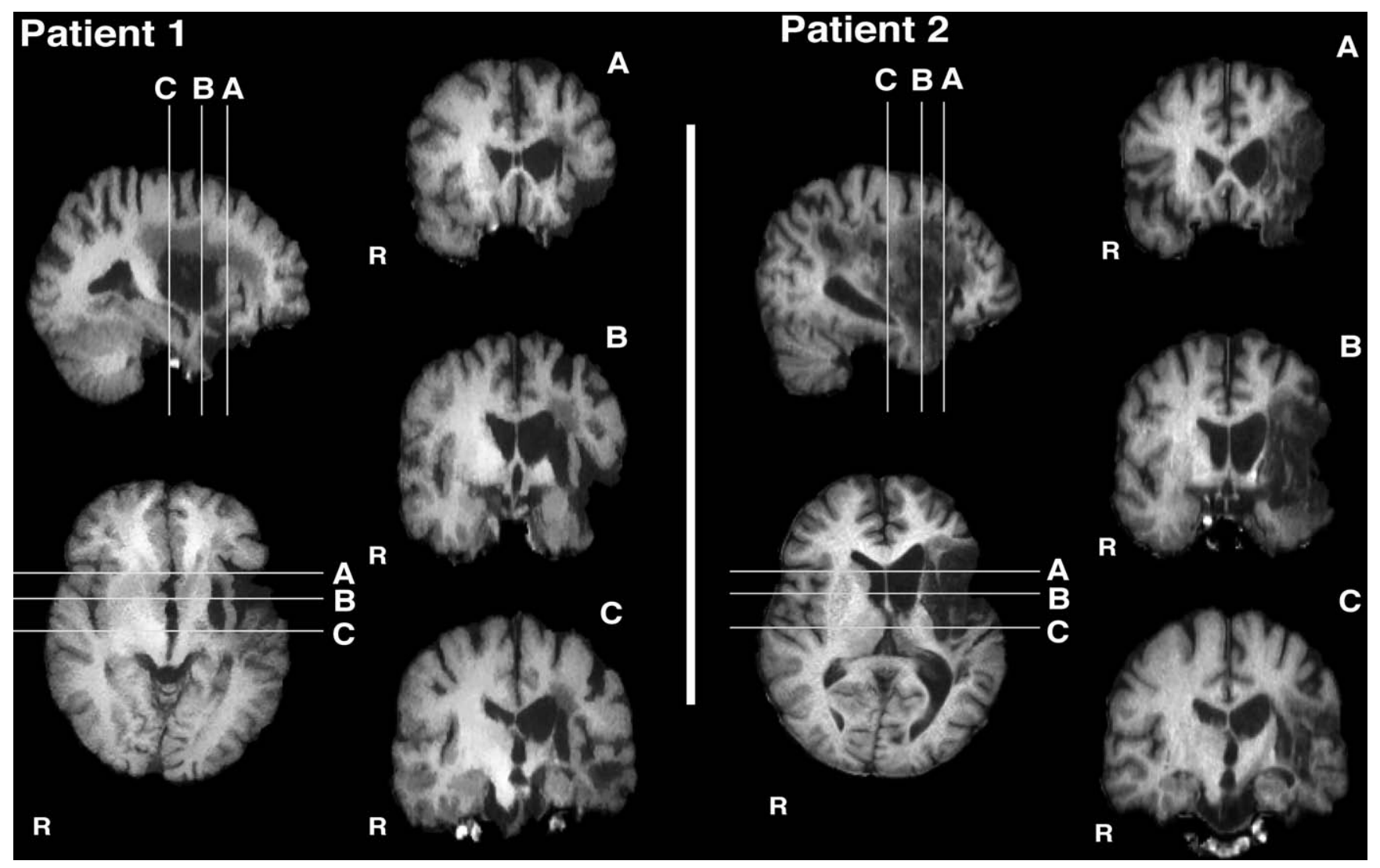

Figure 1. High-resolution T1 images showing the extent of the lesions. Both patients had large left MCA ischemic strokes with lesions encompassing the insular cortex. Patient 1's lesions involved large portions of the insula, parts of SII, basal ganglia, and white matter. Patient 2's lesions were more extensive than patient 1's and involved large portions of the insula and SII, parts of basal ganglia, and white matter. Patient 2 also suffered from hemorrhagic transformation of the ischemic stroke.

determine whether lesioned patients exhibited different sensitivity than healthy control subjects. Since the small number of lesioned subjects $(N=2)$ complicates within-group variance estimates, we also used descriptive statistics using 90 th and 10th percentile range of controls data as additional criteria to determine the significance of sensory alterations displayed by each patient.

Statistical analysis of regional signal changes within the brain Pain-related activations were examined using simple boxcar functions. The regressor was convolved with a gamma-variate model of the hemodynamic response (delay, $6 \mathrm{~s} ; \mathrm{SD}, 3 \mathrm{~s}$ ) and its temporal derivative and was temporally filtered with the same parameters as the fMRI data. For each individual, fixed-effects general linear modeling analyses were used to identify brain activation associated with the modeled hemodynamic response function (Woolrich et al., 2001), whereas random effects analyses were used to assess activation across individuals. $Z$ (Gaussianized T/F) statistic images were thresholded using clusters determined by $z>2.3$ and a (corrected) cluster significance threshold of $p<0.05$ (Worsley et al., 1992).

\section{Region of interest analysis}

To increase statistical power to detect activations within the insular cortex, an insula region of interest (ROI) was used in the analysis to reduce the number of voxels examined to determine whether ipsilateral insular activation (contralateral insular activation not possible because of the extent of the lesions) had occurred when the affected (right) body side was stimulated. The mask was generated from activation data of stimulation of the unaffected (left) side. Additionally, to determine whether there were any differential activations of SI between stimulation of the affected and unaffected sides that may explain altered pain processing, we also examined SI using an ROI analysis.

In the patients, the left SI ROI mask was generated from activation data during stimulation of affected (right) side. This mask was then used to examine the activation within the left SI ROI during stimulation of the affected (right) side. However, no detectible right SI activation was noted during stimulation of the unaffected (left) side. Therefore, to examine right SI activity during the stimulation of the unaffected (left) side, the left SI ROI mask generated from stimulation of the affected (right) side was flipped left-to-right. In the controls, the left and right SI ROI masks were generated from activation data during stimulation of the right and left body sides, respectively. Next, we calculated the left SI/right SI ROI activation ratio. One-factor ANOVA was then used to determine the effects of lesions on this ratio. In addition, patients' data were only considered significantly different from controls if they fell outside of the 90th and 10th percentile range of control subjects' data.

\section{Results \\ Patients}

Radiologic findings

Patient 1 . There were no acute intracranial abnormalities (Fig. 1). There had been a remote segmental left middle cerebral artery infarction with encephalomalacia of the anterior left temporal lobe and portions of the insular cortex including the posterior insula. There was also an infarction involving the left putamen (Fig. 1). These left middle cerebral artery branch infarctions led to brain volume loss as well as associated ex vacuo dilation of the left lateral ventricle and Wallerian degeneration extending into the left cerebral peduncle and pons. Patient 1's lesions involved large portions of the insula, parts of SII, basal ganglia, and white matter (Fig. 1). Minor chronic small vessel ischemic changes were also present in the deep white matter. At the time of the experiment, the time after stroke was 12 years (Table 1 ).

Patient 2. There were no acute intracranial abnormalities (Fig. 1). There was a large remote left middle cerebral artery infarction 
Table 1. Clinical features and tactile thresholds of patients

\begin{tabular}{|c|c|c|c|c|c|c|c|c|}
\hline \multirow[b]{2}{*}{ Subject } & \multirow[b]{2}{*}{ Sex } & \multirow[b]{2}{*}{ Age at onset } & \multirow[b]{2}{*}{ Age at testing } & \multirow[b]{2}{*}{ Time after stroke (years) } & \multicolumn{4}{|c|}{ Tactile thresholds \pm SEM (N) } \\
\hline & & & & & Left arm & Right arm & Left leg & Right leg \\
\hline Patient 1 & M & 41 & 53 & 12 & 1.65 & 1.65 & 3.61 & 3.61 \\
\hline Patient 2 & M & 58 & 59 & 1 & 4.08 & 4.93 & 4.08 & 4.31 \\
\hline Control & $6 \mathrm{M}, 8 \mathrm{~F}$ & $\mathrm{~N} / \mathrm{A}$ & 59 (mean) & $\mathrm{N} / \mathrm{A}$ & $3.15 \pm 0.17$ & $3.12 \pm 0.16$ & $3.61 \pm 0.16$ & $3.62 \pm 0.15$ \\
\hline
\end{tabular}

Patient 1 did not display any tactile deficits compared with the controls. Patient 2's thresholds were slightly higher than those of the controls with thresholds of affected (right) side being slightly higher than those of the unaffected body side. $M$, Male; F, female; N/A, not available.
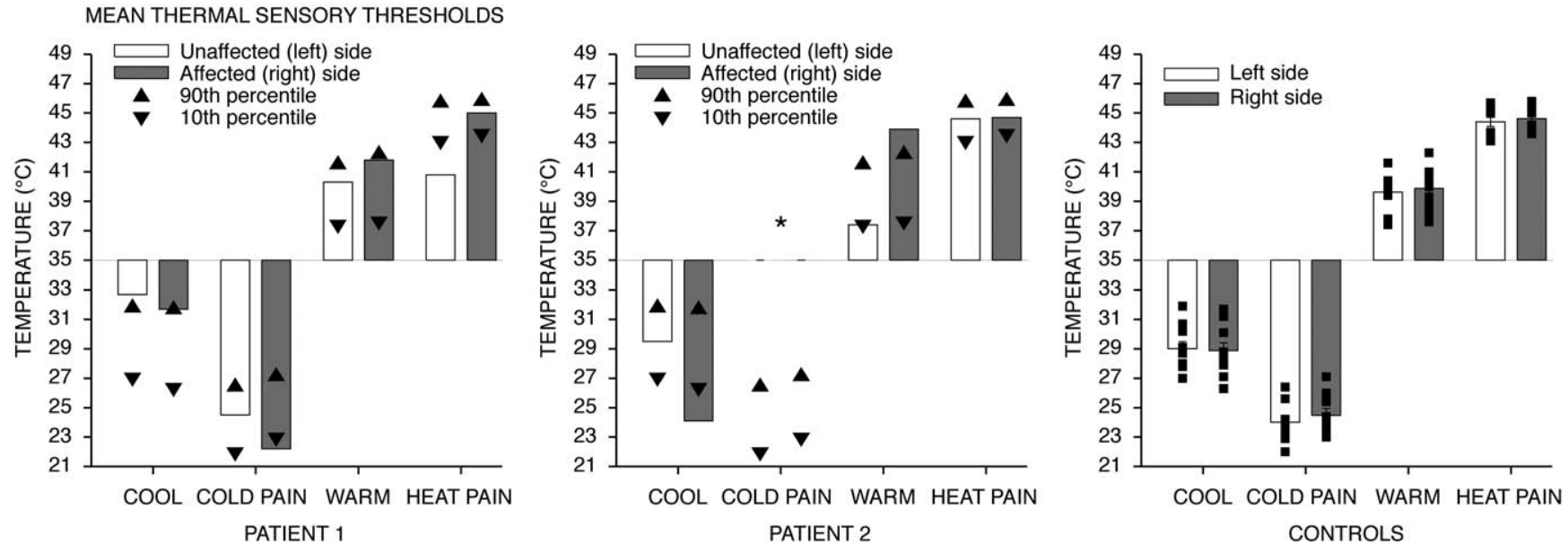

Figure 2. Thermal thresholds (means \pm SEM). Disturbances in temperature sensations seen in patients are consistent with lesions to the insula. In patient 1 , although the heat pain threshold of the affected side was normal, the threshold of the unaffected side was in the range of the innocuous warm detection threshold and is consistent with allodynia. In patient 2 , the innocuous cool detection threshold of the affected side was markedly lower than that of the unaffected side and controls and was in the range of the cold pain threshold. In addition, patient reported feeling no cold pain even at $0^{\circ} \mathrm{C}\left({ }^{*}\right)$. The innocuous warm detection threshold of the affected side was markedly elevated when compared with the unaffected side and controls and was in the range of the heat pain threshold. Both innocuous warm and cool detection thresholds were asymmetric between sides. For each patient, only the average of six measurements per thermal threshold was recorded, so SEM could not be calculated.

with associated ex vacuo dilation of the left lateral ventricle and Wallerian degeneration extending through the internal capsule and into the left cerebral peduncle and left pons. Along the medial margin of this infarction were areas of increased signal on T1weighted images, likely because of cortical laminar necrosis. Patient 2's lesions were more extensive than patient 1's and involved large portions of the insula and SII as well as parts of basal ganglia and white matter (Fig. 1). Diffuse brain volume loss was noted. Note was also made of abnormal increased signal in the distal left internal carotid artery, consistent with severely diminished flow versus complete occlusion of the left internal carotid artery. At the time of the experiment, time after stroke was 1 year (Table 1). It should be noted that patient 2 also suffered from hemorrhagic transformation after his ischemic stroke.

Neurologic findings and tactile thresholds

Patient 1. Sensory examination was normal throughout position and vibration. von Frey testing showed that the patient did not display tactile deficits in any areas tested when compared with those of controls (Table 1). There was residual right hemiparesis: biceps strength was $4 / 5$, wrist flexion and finger flexion were $3+/ 5$ on the Medical Research Council scale. For the right leg, strengths of hip flexors, knee flexors, and extensors were $4+/ 5$. There was no aphasia or impaired cognition. No central poststroke pain (CPSP) was noted. No tremor or spasticity was noted.

Patient 2. There was mild decreased sensation to touch and pin prick of the right face, arm, and leg. von Frey testing showed that, although the patient displayed some tactile deficits, these were relatively mild, suggesting that the patient's perception of touch was mostly intact (Table 1). There was distal weakness involving the right upper extremity. For right upper extremity strength, finger extension was $4-/ 5$, finger flexion was $4+/ 5$, and proximal strength, including the deltoids, was $4+/ 5$. Leg strength was 5/5. There was no aphasia or impaired cognition. No CPSP was noted. No tremor or spasticity was noted.

\section{Thermal thresholds}

Patient 1. The innocuous cool detection thresholds of both affected and unaffected sides were approximately symmetric and were in the range of those of the controls (Fig. 2). The cold pain threshold of the affected (right) body side was slightly lower than that of the unaffected side. However, thresholds of both sides were in the range of those of the controls. The innocuous warm detection threshold of the affected side was slightly higher than that of the unaffected side. The heat pain threshold of the unaffected side was markedly lower than that of the affected side as well as of the controls (Fig. 2). In fact, although the heat pain threshold of the affected side was normal, the heat pain threshold of the unaffected side was in the range of the innocuous warm detection threshold, and is consistent with allodynia.

Patient 2. The innocuous cool detection threshold of the affected side was markedly lower than that of the unaffected side and was in the range of cold pain threshold of the controls (Fig. 2 ). For cold pain, patient 2 did not report feeling any pain even at $0^{\circ} \mathrm{C}$ on both affected and unaffected sides. For innocuous warm detection, the threshold of the unaffected side was normal, but the threshold of affected (right) side was markedly elevated and was in the range of heat pain threshold. Additionally, it is important to note that both innocuous warm and cool detection thresholds in this patient were asymmetric between affected and unaf- 

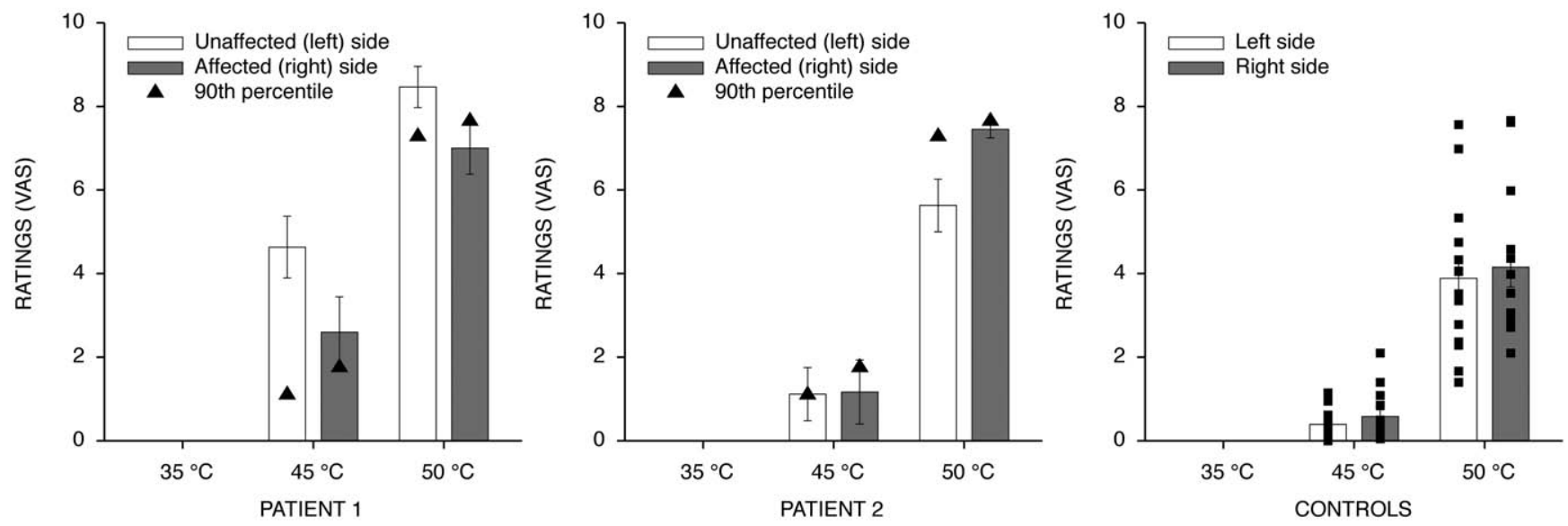

GRADED NOXIOUS HEAT PAIN UNPLEASANTNESS RATINGS
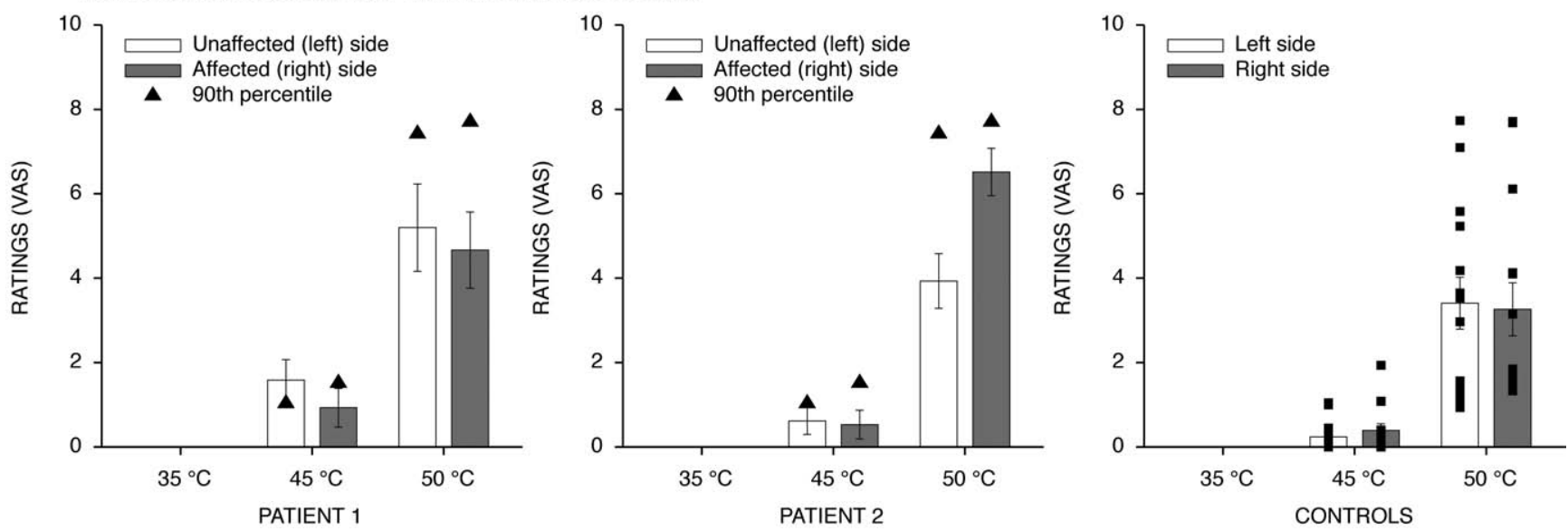

Figure 3. Pain intensity and unpleasantness VAS ratings during the graded noxious stimulation (means \pm SEM). Both patients retained ability to discriminate noxious stimuli of graded intensities. Both exhibited monotonic increases in VAS ratings of pain intensity and unpleasantness as stimulus temperature increased.

fected sides (Fig. 2). In contrast, heat pain thresholds of both affected and unaffected sides were normal and symmetric.

These results (Fig. 2, Table 1) suggest that the lesions likely affected brain areas more involved in temperature sensations than tactile sensations, consistent with the role of the insular cortex in temperature sensation (Craig et al., 2000).

\section{Patients retain the ability to discriminate pain intensity despite lesions}

Both patients were able to evaluate brief noxious stimuli of graded intensities $\left(35,45,50^{\circ} \mathrm{C} ; 5 \mathrm{~s}\right)$ applied to the dorsal calf on affected and unaffected sides. Both patients exhibited monotonic increases in VAS ratings of pain intensity and unpleasantness as stimulus temperature increased (patient 1: pain intensity, $F_{(2,10)}$ $=133.3144, p<0.0001$; pain unpleasantness, $F_{(2,10)}=30.7348$, $p<0.0001$; patient 2: pain intensity, $F_{(2,10)}=172.0429, p<$ 0.0001; pain unpleasantness, $F_{(2,10)}=106.7061, p<0.0001$ ) (Fig. 3 ). No significant effect of body side was observed (patient 1: pain intensity, $F_{(1,5)}=3.7824, p=0.1094$; pain unpleasantness, $F_{(1,5)}$ $=0.4547, p=0.5300$; patient 2: pain intensity, $F_{(1,5)}=4.7348$, $p=0.0815$; pain unpleasantness, $\left.F_{(1,5)}=1.4650, p=0.2802\right)$ (Fig. 3). These results suggest that patients' ability to discriminate pain intensity still remained intact despite large unilateral lesions of the insula. During graded noxious stimulation of the controls, subjects exhibited monotonic increases in VAS ratings of pain intensity and unpleasantness as stimulus temperature increased (intensity, $F_{(2,12)}=34.6503, p<0.0001$; unpleasantness, $F_{(2,12)}=$ $19.1711, p=0.0002$ ) (Fig. 3). There was no significant main effect of body sides on pain intensity and unpleasantness ratings (intensity, $F_{(1,13)}=3.3841, p=0.0888$; unpleasantness, $F_{(1,13)}=$ $0.0013, p=0.9714$ ) (Fig. 3).

\section{Elevated pain ratings in patients with insular lesions}

During long-duration noxious stimulation (30 s on, 30 s off; five cycles; $49^{\circ} \mathrm{C}$ ) during the fMRI session, both patients exhibited significantly higher VAS ratings of pain intensity of the affected (right) body side compared with those of the control group $\left(F_{(1,13)}=7.3720 ; p=0.0177\right)$ (Fig. $4 A$ ). Interestingly, both patients' pain unpleasantness ratings of the affected body side did not significantly differ from those of the control group, although patient 2 displayed a trend toward elevated pain unpleasantness on the affected side $\left(F_{(1,13)}=2.3825 ; p=0.1467\right)$ (Fig. $\left.4 B\right)$. Both patients' pain intensity and unpleasantness ratings of the unaffected (left) body side did not significantly differ from those of the control group (pain intensity, $F_{(1,13)}=3.0652, p=0.1035$; pain unpleasantness, $F_{(1,13)}=0.0950, p=0.7629$ ) (Fig. 4), although patient 1's pain intensity ratings during both short- and longduration stimulation of unaffected (left) body side were higher than any of the individual control subjects (Figs. 3, 4A).

Each patient responded very differently during long duration noxious stimulation. Patient 1 showed markedly elevated pain intensity ratings across both body sides. In fact, patient 1's pain 

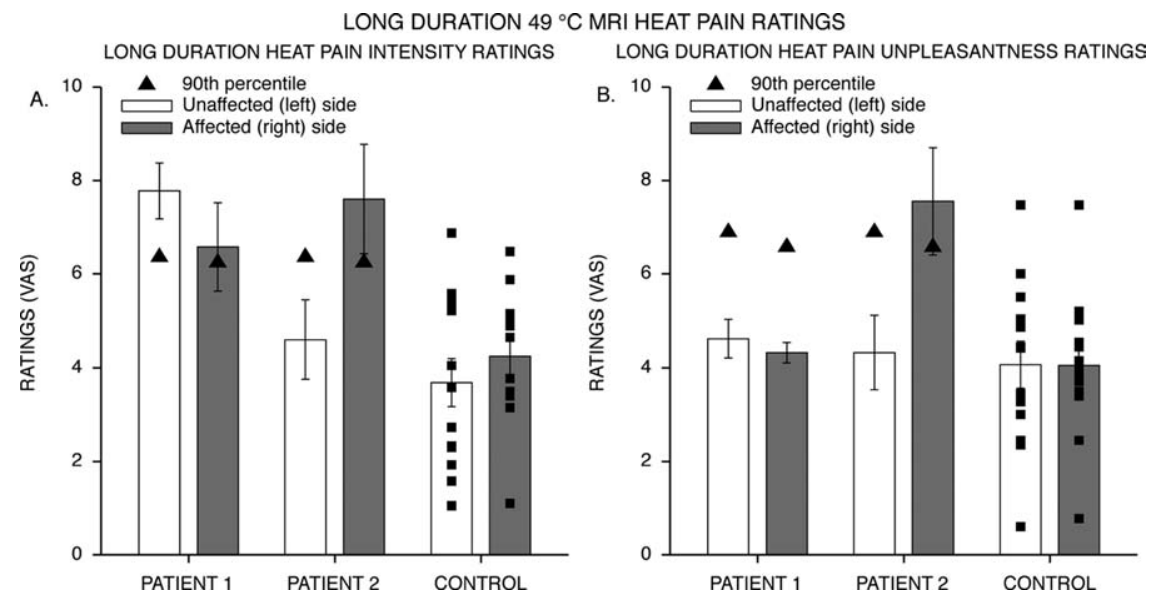

Figure 4. Pain intensity and unpleasantness VAS ratings during the long-duration noxious stimulation (means \pm SEM). The solid squares indicate individual control's data. The solid triangles indicate 90 th percentile of the control's data. Both patients exhibited significantly elevated pain intensity ratings compared with those of the control group $(\boldsymbol{A})$. However, the effect of lesion on pain unpleasantness was not statistically significant, although patient 2 displayed a trend toward elevated pain unpleasantness on the affected side $(\boldsymbol{B})$. Additionally, each patient responded differently. Whereas patient 2 had asymmetric pain ratings between sides without much disparity between the two pain dimensions, patient 1 had elevated pain intensity ratings across sides with normal pain unpleasantness.

intensity ratings of both body sides were higher than any of the individual control subjects (Fig. 4A). More interestingly, despite significantly elevated pain intensity ratings, patient 1 exhibited relatively normal pain unpleasantness ratings across both body sides (Fig. $4 B$ ). These results suggest that an altered pain affect processing and a disconnect between the two dimensions of pain may have occurred. Patient 2, however, displayed elevated pain intensity and unpleasantness ratings on the affected without much disparity between the two dimensions of pain (Fig. 4). In fact, both pain intensity and unpleasantness ratings of the affected body side of patient 2 were higher than any of the individual control subjects. Nevertheless, although both patients responded differently, elevated pain ratings and hypersensitivity, in general, were noted in both patients.

\section{Asymmetry of pain ratings between affected and unaffected body sides}

To determine whether there are significant differences in pain ratings between the two sides, we examined pain intensity and pain unpleasantness ratings between affected and unaffected sides within each patient. During long-duration noxious stimulation, patient 1's pain intensity and unpleasantness ratings did not significantly differ between affected and unaffected sides (pain intensity, $F_{(1,3)}=3.9452, p=0.1412$; pain unpleasantness, $F_{(1,3)}=0.60, p=0.4950$ ) (Fig. 4). In patient 2, both pain intensity and unpleasantness ratings were significantly greater on the affected side than they were on the unaffected side (pain intensity, $F_{(1,3)}=11.4165, p=0.0431$; pain unpleasantness, $F_{(1,3)}=$ $13.15491, p=0.0361$ ) (Fig. 4). In the control group, there was no difference in pain intensity and unpleasantness ratings of longduration noxious stimuli applied to left and right sides (intensity, $F_{(1,12)}=2.8813, p=0.1154$; unpleasantness, $F_{(1,12)}=0.0053, p=$ 0.9429) (Fig. 4). This suggests that, although some disturbances in pain and temperature sensations were shared by both patients, other aspects of pain processing may be differentially altered in each patient because of differences in their lesions and other factors unique to each individual.

\section{Activations of the insula and other pain-related activations}

During long-duration noxious stimulation $\left(49^{\circ} \mathrm{C}\right.$; $30 \mathrm{~s}$ on, $30 \mathrm{~s}$ off; five cycles $)$ of the dorsal calf of the unaffected (left) body side in both patients, pain-induced brain activations were identified within the ACC, supplementary motor area (SMA), SII, insula, dorsolateral prefrontal cortex (DLPFC), and cerebellum (Fig. 5, Table 2). However, it is important to note that, although patient 1's ACC activation was mostly within the right hemisphere, patient 2's ACC activation appears to be mostly within the left hemisphere. No thalamic activations were detected in either patient. Stimulation of the left side in controls activated ACC, SMA, SII, insula, SI, thalamus, and cerebellum (Fig. 5, Table 2). These patterns of activations are consistent with normal brain activations during pain (Coghill et al., 1994, 2001; Peyron et al., 2000; Oshiro et al., 2007). In the control group, insular activations were detected bilaterally. In the patient group, because the left insula was heavily damaged, only the right insula could have been activated (Fig. 5, Table 2). On stimulation of the unaffected (left) body side, contralateral (right) insular activation was detected in both patients.

During painful stimulation of the affected (right) body side in patient 1, pain-induced brain activations were identified within the SMA, SI, SII, DLPFC, the frontal operculum, and the cerebellum (Fig. 5, Table 2). In patient 2, stimulation of the right (affected) leg activated SI, SII, SMA, cerebellum, ACC, and the contralateral (left) thalamus (Fig. 5, Table 2). It is interesting to note that patient 2 displayed a robust activation of the contralateral (left) thalamus, although no ipsilateral thalamic activation was detected. Nevertheless, neither patient displayed any ipsilateral (right) insular activation (Fig. 5, Table 2). In both patients, only the ipsilateral (right) insula could be activated because of damage to the contralateral (left) insula. During stimulation of the right dorsal calf of the control group, pain activations detected were similar to those during stimulation of the left leg and included bilateral insular activation (Fig. 5, Table 2). In addition, insular ROI analysis was used to examine the possibility of subthreshold activation of the insula in patients. ROI analysis of the insula showed that stimulation of the unaffected body side in both patients produced a detectable contralateral insular activation within the insular ROI, whereas stimulation of the affected body side still did not produce any detectable activation within the ipsilateral insula. This suggests that insular activation may not be necessary for a conscious experience of pain, and that contralateral insular activation appears to be necessary to produce ipsilateral insular activation.

\section{Differences in SI activation between lesioned subjects and healthy controls}

During stimulation of the unaffected (left) body side in both patients, there were no detectable SI activations (Fig. 6, Table 2). In contrast, stimulation of the affected (right) body side produced robust contralateral SI activations in both lesioned subjects (Fig. 6, Table 2). It appears that SI activations in patients only became sufficiently large to be reliably detected during stim- 


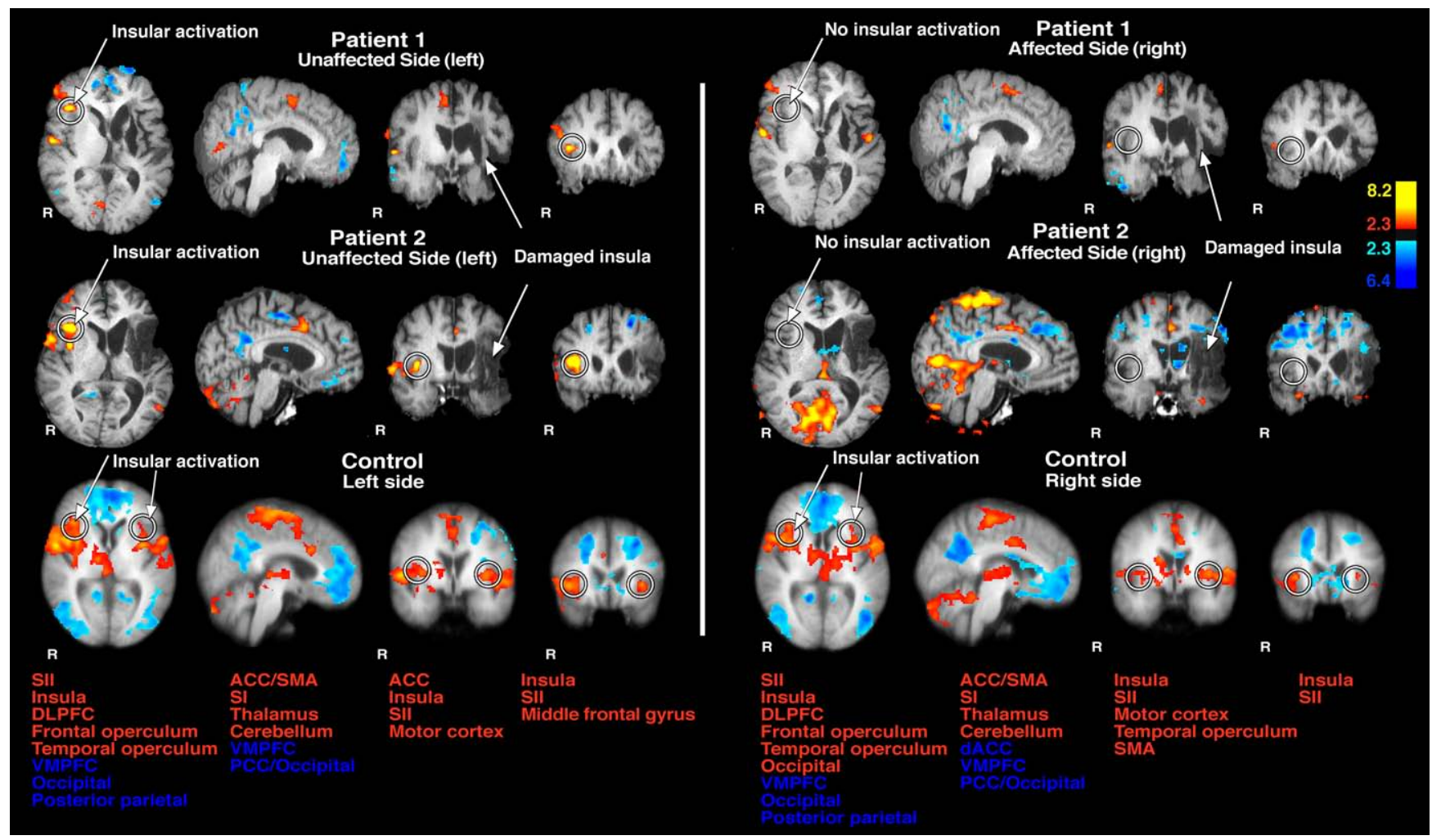

Figure 5. Pain-related activation of the insula. Stimulation of the unaffected (left) leg in patients activated similar brain areas as in controls. However, in the patients only the right (contralateral) insula was activated since the left insula was damaged by the lesions. In the controls, insular activation was detected bilaterally. In contrast, painful stimulation of the affected (right) leg in patients did not produce any detectable right (ipsilateral) insular activation. This suggests that insular activation may not be necessary for a conscious experience of pain and that contralateral insular activation appears to be necessary to produce ipsilateral insular activation. The structures named at the bottom are for both controls and patients. VMPFC, Ventromedial prefrontal cortex; PCC, posterior cingulate cortex; dACC, dorsal anterior cingulate cortex.

ulation of the affected body side when insular activation was not present (Figs. 5, 6; Table 2). Stimulation of the either side in controls generated reliable contralateral SI activation.

SI ROI analysis was used to determine whether these differences in contralateral SI activation between stimulation of affected and unaffected sides were significantly different from healthy controls. Both patients' left/right SI ROI activation ratios were significantly greater than those of the controls $\left(F_{(1,13)}=\right.$ 12.5121; $p=0.0036$ ) (Fig. 7). In fact, both patients' ratios were greater than any of the individual control subjects (Fig. 7). These results suggest that, in the absence of the insula, brain areas such as SI may be recruited to help with the processing of nociceptive information.

\section{Recruitment of DLPFC activation in lesioned subjects}

Right DLPFC activations during painful stimulation were detected in both patients (Fig. 8, Table 2). Painful stimulation of the unaffected (left) body side activated the right DLPFC in both patient 1 and patient 2 but not in the controls (Fig. 8, Table 2). However, stimulation of the affected (right) body side activated the right DLPFC in patient 1 , but not in patient 2 or controls (Fig. 8 , Table 2). Although DLPFC activation has been reported during spatial discrimination of pain (Oshiro et al., 2007), it was not detected in the controls in this investigation. Right DLPFC activations seen in our patients may suggest increased burden on the remaining neural networks to process nociceptive information in the face of insular damage.

\section{Discussion}

Although the insular cortex has been frequently shown to be activated during the processing of pain, the specific roles that this structure can play in the generation of a pain experience remain poorly characterized. Stimulus-response studies suggest that it may be positively related to the perceived magnitude of pain and, accordingly, may be involved in sensory-discriminative processing (Derbyshire et al., 1997; Coghill et al., 1999). However, both patients retained the ability to provide ratings of graded pain intensity during noxious stimulation of their affected side despite having large areas of the insular cortex damaged by strokes and exhibiting no detectible activity in either the contralateral or ipsilateral insular cortex. In sharp contrast to the diminished experience of pain that would be predicted from previous studies of insular lesions, both patients exhibited significantly increased ratings of pain intensity. When taken together with the insular activation detected in studies that evoke analgesia by either pharmacological or psychological manipulations, this suggests that the insula may instead play a complex, modulatory role in the processing of nociceptive information (Petrovic et al., 2002; Lorenz et al., 2003; Derbyshire et al., 2004; Koyama et al., 2005; Zubieta et al., 2005; Kong et al., 2006, 2007; Craggs et al., 2007). Moreover, during stimulation of the affected sides, both lesion patients exhibited consistently greater activation of SI than during stimulation of the unaffected side (relative to healthy controls) that may reflect a recruitment of additional SI activity to compensate for the loss of the contribution of the insula to sensory processing. These findings indicate that insular activation, 
Table 2. Pain-related brain activations

\begin{tabular}{|c|c|c|c|c|c|c|}
\hline \multirow[b]{2}{*}{ Region } & \multicolumn{2}{|c|}{ Patient 1's side of stimulation } & \multicolumn{2}{|c|}{ Patient 2's side of stimulation } & \multicolumn{2}{|l|}{ Controls' side of stimulation } \\
\hline & $\mathrm{L}$ & $\mathrm{R}$ & $\mathrm{L}$ & $\mathrm{R}$ & $\mathrm{L}$ & $\mathrm{R}$ \\
\hline \multicolumn{7}{|l|}{ Cerebellum } \\
\hline Right & - & $3.62(240 ; 50,-76,-16)$ & $4.73(2254 ; 26,-62,-28)$ & $6.97(11,952 ; 34,-70,-20)$ & $3.717(9128 ; 6,-84,-34)$ & $4.17(5021 ; 20,-88,-34)$ \\
\hline Left & $3.9(408 ;-38,-60,-36)$ & $4.39(342 ;-16,-72,-40)$ & $4.02(2254 ;-48,-64,-24)$ & $5.16(592 ;-28,-44,-50)$ & $4.089(9128 ;-30,-70,-26)$ & $4.03(5021 ;-26,-76,-30)$ \\
\hline \multicolumn{7}{|l|}{ Thalamus } \\
\hline Right & - & - & - & - & $3.559(9128 ; 4,-24,4)$ & $3.272(5963 ; 10,-4,4)$ \\
\hline Left & - & - & - & $3.489(11,952 ;-2,-16,6)$ & - & $3.610(5963 ;-12,-14,-4)$ \\
\hline \multicolumn{7}{|l|}{ Globus pallidus/putamen } \\
\hline Right & - & - & - & - & $3.135(9128 ; 16,10,0)$ & $3.245(5963 ; 18,-2,-8)$ \\
\hline Left & - & - & - & - & $3.453(9128 ;-30,0,2)$ & $3.614(5963 ;-18,-20,12)$ \\
\hline \multicolumn{7}{|l|}{ Anterior insula } \\
\hline Right & $6.48(2003 ; 42,28,6)$ & - & $7.64(1286 ; 34,22,6)$ & - & $5.21(9128 ; 38,8,8)$ & $4.47(5963 ; 36,26,0)$ \\
\hline Left & - & - & - & - & $4.47(2155 ;-36,4,6)$ & $4.08(5963 ;-34,12,-2)$ \\
\hline \multicolumn{7}{|l|}{ Posterior insula } \\
\hline Right & - & - & - & - & $3.961(9128 ; 38,-18,12)$ & - \\
\hline Left & - & - & - & - & - & $3.132(5963 ;-32,-18,8)$ \\
\hline \multicolumn{7}{|l|}{ Prefrontal cortex } \\
\hline Right & $3.949(2003 ; 44,42,2)$ & $3.7(435 ; 52,46,0)$ & $4.22(555 ; 42,52,0)$ & - & - & - \\
\hline Left & - & - & $2.61(188 ;-38,54,12)$ & - & - & - \\
\hline \multicolumn{7}{|l|}{ Anterior cingulate cortex } \\
\hline Right & $2.52(169 ; 12,2,48)$ & $3.463(239 ; 6,10,56)$ & - & - & $4.21(2245 ; 4,16,30)$ & $2.891(1774 ; 6,10,34)$ \\
\hline Left & - & - & $4.32(216 ;-2,6,38)$ & $5.195(11,952 ;-10,-4,40)$ & $3.682(2245 ;-4,8,38)$ & $3.254(1774 ;-6,2,38)$ \\
\hline \multicolumn{7}{|l|}{ IPL/SPL } \\
\hline Right & - & - & $3.06(417 ; 64,-36,36)$ & $3.308(11,952 ; 8,-54,62)$ & $3.805(9128 ; 54,-38,42)$ & $3.25(650 ; 54,-40,28)$ \\
\hline Left & - & $4.54(417 ;-22,-54,64)$ & - & - & - & $3.258(1774 ;-22,-42,62)$ \\
\hline SI (leg) & - & $3.38(417 ;-16,-38,66)$ & - & $6.052(11,952 ;-16,-26,74)$ & $4.06(2245 ; 4,-34,74)$ & $4.03(1774 ;-10,-24,72)$ \\
\hline \multicolumn{7}{|l|}{ SII } \\
\hline Right & $4.71(2003 ; 68,-12,18)$ & $3.12(255 ; 52,-10,10)$ & $5.44(417 ; 66,-24,22)$ & $6.75(597 ; 66,-26,18)$ & $4.65(9128 ; 66,-34,22)$ & $3.71(650 ; 62,-22,16)$ \\
\hline Left & - & $4.22(151 ;-60,-24,18)$ & - & - & $4.05(2155 ;-66,-22,16)$ & $4.183(5963 ;-56,6,6)$ \\
\hline \multicolumn{7}{|l|}{ Supplementary motor area } \\
\hline Right & $3.48(169 ; 6,6,58)$ & $3.74(239 ; 6,0,60)$ & - & $4.61(663 ; 22,12,62)$ & $4.84(2245 ; 8,-16,72)$ & $3.130(1774 ; 0,2,50)$ \\
\hline Left & - & $3.81(192 ;-12,-22,62)$ & $4.096(216 ;-4,12,40)$ & $6.542(11,952 ;-6,-32,68)$ & $3.518(2245 ;-6,-2,64)$ & $5.02(1774 ;-10,-14,64)$ \\
\hline \multicolumn{7}{|l|}{ 〈Deactivation〉 } \\
\hline Posterior cingulate cortex & $-3.89(1280 ; 8,-70,22)$ & $-4.03(1152 ; 10,-56,18)$ & $-6.42(1175 ; 2,-58,28)$ & $-4.60(902 ; 2,-38,26)$ & $-4.53(27,536 ;-6,-58,16)$ & $-4.029(14,209 ; 6,-52,30)$ \\
\hline VMPFC & $-5.33(2123 ;-6,60,10)$ & - & $-4.62(898 ; 8,54,18)$ & $-3.708(9488 ; 14,64,16)$ & $-4.036(27,536 ;-12,58,10)$ & $-4.53(8370 ; 2,56,-6)$ \\
\hline Occipital lobe & $-4.29(257 ;-50,-72,8)$ & $-4.79(1152 ; 6,-62,20)$ & - & - & $-3.907(27,536 ; 40,-74,28)$ & $-4.118(14,209 ;-28,-78,30)$ \\
\hline
\end{tabular}

Peak Z scores for the controls were obtained from group analysis. Cluster sizes and peak locations are listed within parentheses as the number of voxels and $x, y, z$ coordinates (in millimeters) according to standard stereotaxic space. L, Left; $\mathrm{R}$, right; IPL, Inferior parietal lobule; SPL, superior parietal lobule; - , no statistically reliable change.

although frequently observed in studies of pain, may not be necessary to elicit a conscious pain experience. Thus, subjective awareness of pain intensity can be realized via multiple, distinct patterns of brain activity.

\section{Specificity of insular lesions}

Systematic studies of patients with brain lesions affecting the insular cortex have been rarely performed because of the difficulty of recruiting individuals who are MRI compatible, free from central poststroke pain, and do not exhibit aphasia. Furthermore, the interpretation of lesion studies is complicated by the uniquely individual patterns of damage. However, when commonalities between individuals are related to overlapping regions of damage, substantial insights into the function of the damaged region can be derived. In the present investigation, the lesions affected large portions of the insular cortex with patient 2's lesions being more extensive than patient 1's. Despite the differences in the extent of each individual's lesion, both individuals consistently reported abnormally high sensitivity to pain and exhibited marked increases in the activity of the left primary somatosensory cortex (ipsilateral to the lesioned insula). These common findings in both patients further imply that damaged brain areas common to both patients may be responsible.

Although both patients' lesions involved structures outside the insular cortex such as the basal ganglia and white matter tracts, the disturbances in temperature sensations that both pa- tients exhibited are consistent with the damage to the insula because this structure is thought to be involved in the processing of temperature sensations (Craig et al., 2000). Neither patient displayed motor symptoms such as spasticity or tremors that would be characteristic of basal ganglia lesions. In fact, ongoing studies show reduced pain sensitivity in patients with basal ganglia lesions in contrast to the increased pain sensitivity observed in the present investigation (our unpublished observations).

We also selected the lower legs as sites of stimulation because the SI leg representations were far removed from brain areas affected by the strokes. Mild tactile deficits in our patients suggest that SI and thalamocortical projections to SI were minimally affected by the strokes because lesions affecting SI can significantly disrupt tactile sensation (Knecht et al., 1996). Furthermore, stimulation of the affected leg in both patients generated pronounced activation of SI. These findings suggest that the psychophysical differences seen in our study were primarily attributable to lesions affecting the insula, and did not result from either direct damage to SI or from disruption of thalamocortical projections to SI.

Although lesions affected some portions of SII and posterior insula, we did not see hypoalgesia, as expected of SII and posterior insular lesions (Greenspan and Winfield, 1992; Greenspan et al., 1999). Thus, these results may likely be attributable to damage that also involves the anterior insula. Although the functions of posterior and anterior insula differ because of their distinct ana- 


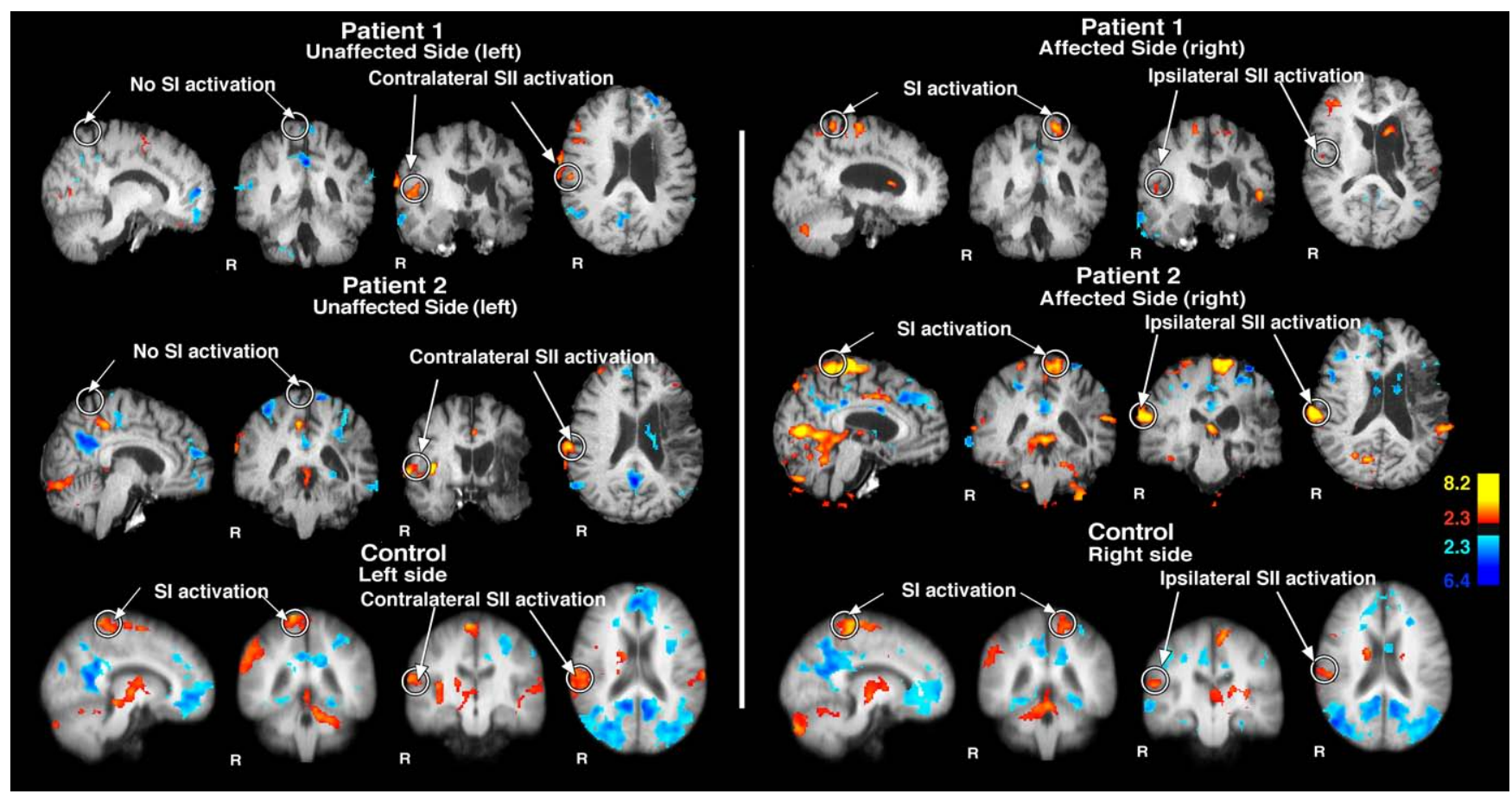

Figure 6. Sl activation during pain. Stimulation of the unaffected (left) side did not generate any Sl activation in both patients. Interestingly, stimulation of the affected (right) body side produced robust contralateral SI activations in both patients. It appears that $\mathrm{SI}$ activations in patients may only be reliably detected during stimulation of the affected side when insular activation was not present (Fig. 5). These results suggest SI may be recruited to help with processing of nociceptive information after insular damage.

tomical connections, both divisions are extensively interconnected (Mesulam and Mufson, 1982; Mufson and Mesulam, 1982; Friedman et al., 1986). Thus, damage to either division may disrupt the transfer of information and the function of both divisions of the insula. Nevertheless, ascribing the alterations in pain sensitivity to the anterior insula should be done with caution because portions of both anterior and posterior insula were damaged.

\section{Altered sensory processing and insular lesions}

To date, little is known about how insular lesions alter the processing of suprathreshold noxious stimuli. Previous studies using sensory testing indicate that pain thresholds are elevated after lesions involving the posterior insula and parietal operculum, but not the anterior insula (Greenspan and Winfield, 1992; Greenspan et al., 1999). During testing with suprathreshold stimuli, individuals with insular lesions have been reported to exhibit pain asymbolia, a reduced appreciation of the meaning and significance of noxious stimuli while retaining the capacity to identify such stimuli as painful (Berthier et al., 1988). Although the partial dissociation of pain intensity and unpleasantness observed in patient 1 may be consistent with reports of pain asymbolia, the view that insular lesions preferentially alter cognitive/ emotional appreciation of pain may be oversimplified. In response to long-duration noxious stimuli, both lesion subjects in the present investigation exhibited pain sensitivity that was substantially higher than age-matched control subjects, but neither had ratings of pain unpleasantness that were significantly below normal.

In contrast to other studies that show strong insular activation without SI activation (Derbyshire et al., 1994; Tölle et al., 1999; Peyron et al., 2000), the absence of insular contribution to nociceptive processing appears to be associated with a substantial increase in SI activation during nociceptive stimulation. Thus, SI

\section{LEFT/RIGHT SI ROI ACTIVATION RATIO}

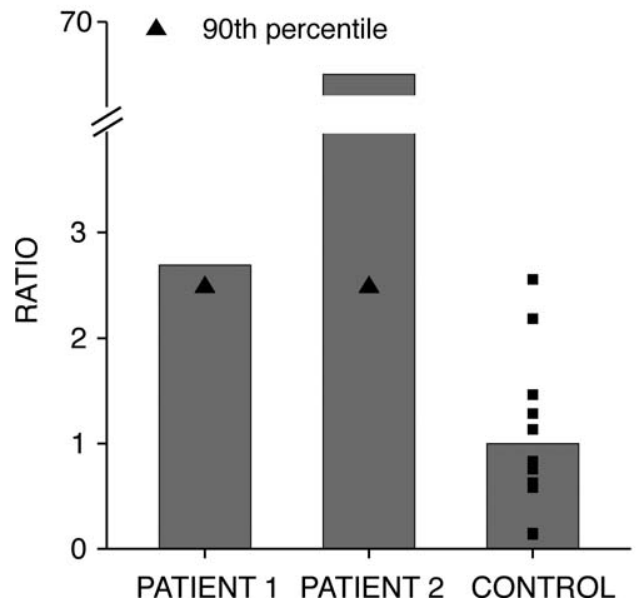

Figure 7. Left/right SI ROI activation ratio. The solid squares indicate individual control's data. The solid triangles indicate 90th percentile of the control's data. In both patients, the left/right SI ROI activation ratios were significantly greater than those of the controls. Thus, contralateral SI activation during stimulation of the affected (right) side relative to contralateral Sl activation during stimulation of the unaffected (left) side was significantly greater in patients than the controls. This suggests that, in the absence of insula, brain areas such as SI may be recruited to help with the processing of nociceptive information.

and its associated networks may be recruited to help with processing of nociceptive information after insular damage. Additionally, right DLPFC activation noted in our patients may represent processes related to the evaluation of nociceptive information, reflecting increased demands on the remaining neural networks after insular damage. These findings suggest that there are multiple ways that the brain can process nociceptive information and instantiate an experience of pain. 


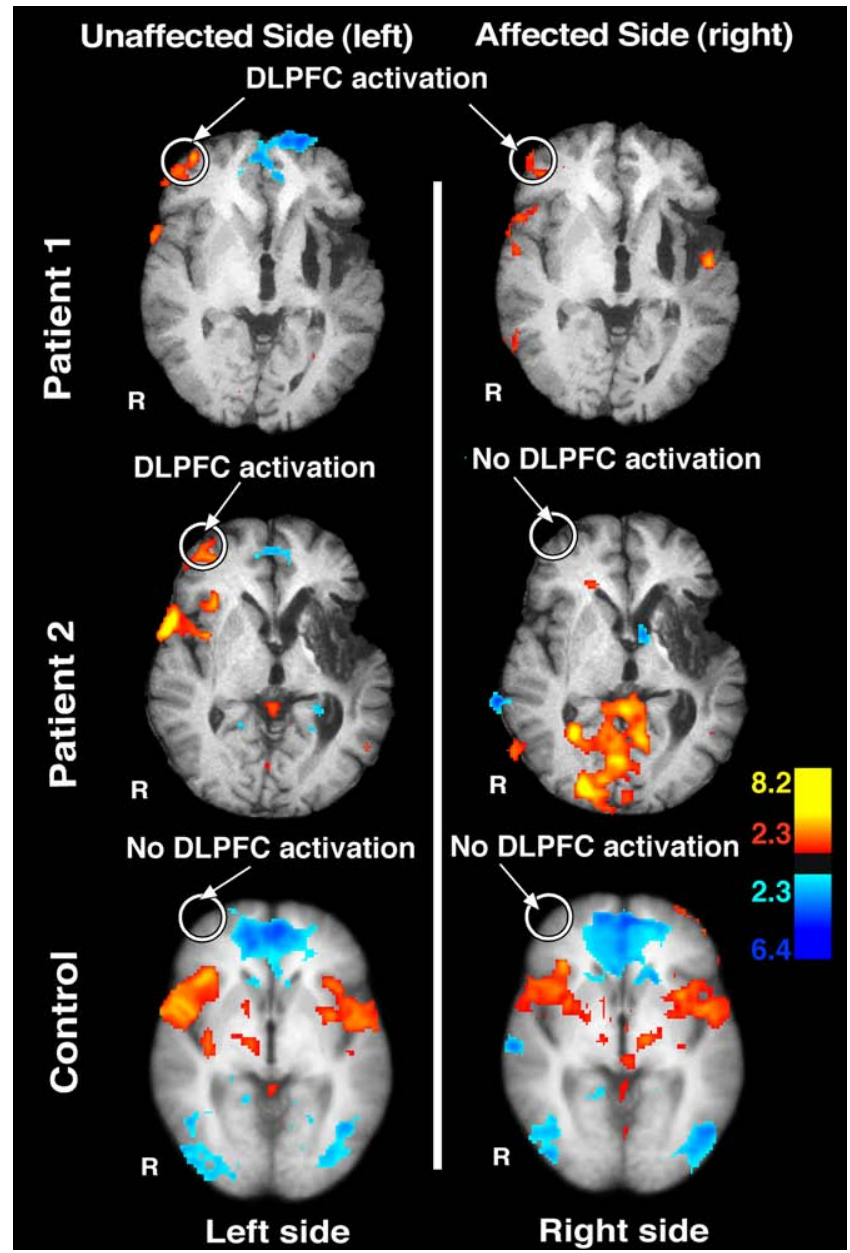

Figure 8. DLPFC activation during pain. Right DLPFC activations during painful stimulation were detected in both patients, but not in the controls. Painful stimulation of the unaffected (left) side activated the right DLPFC in both patients but not in the controls. However, stimulation of the affected (right) body side activated the right DLPFC in patient 1 , but not in patient 2 and controls. Right DLPFC activations may represent recruitment of additional brain areas to help with processing nociceptive information in the face of insular damage.

\section{Top-down signal modulation and elevation of pain ratings after insular lesions}

Both patients exhibited increased pain ratings relative to those of normal controls. Such increases in pain sensitivity may be indicative of loss of descending inhibitory control, because the insular cortex is connected to regions such as the ACC and DLPFC (Mesulam and Mufson, 1982; Mufson and Mesulam, 1982) that are associated with activation of the periaqueductal gray (Lorenz et al., 2003; Gebhart, 2004; Ohara et al., 2005; Kong et al., 2007; Tracey and Mantyh, 2007). However, converging lines of evidence from expectation, hypnosis, and placebo studies suggest that the insula may play a role in pain modulation by tuning the responsiveness of other brain areas via corticocortical interactions (Petrovic et al., 2002; Lorenz et al., 2003; Derbyshire et al., 2004; Koyama et al., 2005; Zubieta et al., 2005; Kong et al., 2006, 2007; Craggs et al., 2007). Complex cognitive information related to mood, previous experience, expectation, and emotion may flow from various brain networks involving the amygdala, hippocampus, ACC, and prefrontal cortex to the anterior insula to be integrated with nociceptive information (Mufson et al., 1981; Mesulam and Mufson, 1982; Mufson and Mesulam, 1982; Friedman and Murray, 1986; Friedman et al., 1986). In the environ- mental context of an experimental setting, the insula may encode a safety signal that tunes afferent sensory processing mechanisms to reflect the previous information that the noxious stimulus is safe and well controlled and will not result in long-term damage. These interactions may contribute importantly to modulation of pain affect and pain intensity processing in a context-relevant manner. This ability to use internal knowledge to modulate the gain of incoming information through top-down signal modulation may be disrupted by insular lesions. As a result, pain ratings were elevated.

\section{Conclusion}

The insula is well positioned to both send and receive information from areas important in sensory processing as well as memory retrieval, attention, and affect (Mufson et al., 1981; Mesulam and Mufson, 1982; Mufson and Mesulam, 1982; Friedman and Murray, 1986; Friedman et al., 1986). This dynamic, bidirectional interactive network provides a substrate for the insula to integrate higher-level internal cognitive information with incoming afferent sensory information. Moreover, the insula may selectively gate nociceptive information at the cortical level to modulate varying levels of appreciation of the stimulus. Through using complex cognitive information to provide modulation of cognitive-evaluative, affective, and sensory discriminative dimensions of pain, the insula contributes to the construction of a unique signature/fingerprint of pain experience for each individual.

\section{References}

Berthier M, Starkstein S, Leiguarda R (1988) Asymbolia for pain: a sensorylimbic disconnection syndrome. Ann Neurol 24:41-49.

Coghill RC, Talbot JD, Evans AC, Meyer E, Gjedde A, Bushnell MC, Duncan GH (1994) Distributed processing of pain and vibration by the human brain. J Neurosci 14:4095-4108.

Coghill RC, Sang CN, Maisog JM, Iadarola MJ (1999) Pain intensity processing within the human brain: a bilateral, distributed mechanism. J Neurophysiol 82:1934-1943.

Coghill RC, Gilron I, Iadarola MJ (2001) Hemispheric lateralization of somatosensory processing. J Neurophysiol 85:2602-2612.

Craggs JG, Price DD, Verne GN, Perlstein WM, Robinson MM (2007) Functional brain interactions that serve cognitive-affective processing during pain and placebo analgesia. Neuroimage 38:720-729.

Craig AD, Chen K, Bandy D, Reiman EM (2000) Thermosensory activation of insular cortex. Nat Neurosci 3:184-190.

Derbyshire SW, Jones AK, Devani P, Friston KJ, Feinmann C, Harris M, Pearce S, Watson JD, Frackowiak RS (1994) Cerebral responses to pain in patients with atypical facial pain measured by positron emission tomography. J Neurol Neurosurg Psychiatry 57:1166-1172.

Derbyshire SW, Jones AK, Gyulai F, Clark S, Townsend D, Firestone LL (1997) Pain processing during three levels of noxious stimulation produces differential patterns of central activity. Pain 73:431-445.

Derbyshire SW, Whalley MG, Stenger VA, Oakley DA (2004) Cerebral activation during hypnotically induced and imagined pain. Neuroimage 23:392-401.

Friedman DP, Murray EA (1986) Thalamic connectivity of the second somatosensory area and neighboring somatosensory fields of the lateral sulcus of the macaque. J Comp Neurol 252:348-373.

Friedman DP, Murray EA, O’Neill JB, Mishkin M (1986) Cortical connections of the somatosensory fields of the lateral sulcus of macaques: evidence for a corticolimbic pathway for touch. J Comp Neurol 252:323-347.

Gebhart GF (2004) Descending modulation of pain. Neurosci Biobehav Rev 27:729-737.

Greenspan JD, Winfield JA (1992) Reversible pain and tactile deficits associated with a cerebral tumor compressing the posterior insula and parietal operculum. Pain 50:29-39.

Greenspan JD, Lee RR, Lenz FA (1999) Pain sensitivity alterations as a function of lesion location in the parasylvian cortex. Pain 81:273-282. 
Jenkinson M, Bannister P, Brady M, Smith S (2002) Improved optimization for the robust and accurate linear registration and motion correction of brain images. Neuroimage 17:825-841.

Knecht S, Kunesch E, Schnitzler A (1996) Parallel and serial processing of haptic information in man: effects of parietal lesions on sensorimotor hand function. Neuropsychologia 34:669-687.

Kong J, Gollub RL, Rosman IS, Webb JM, Vangel MG, Kirsch I, Kaptchuk TJ (2006) Brain activity associated with expectancy-enhanced placebo analgesia as measured by functional magnetic resonance imaging. J Neurosci 26:381-388.

Kong J, Kaptchuk TJ, Polich G, Kirsch I, Gollub RL (2007) Placebo analgesia: findings from brain imaging studies and emerging hypotheses. Rev Neurosci 18:173-190.

Koyama T, McHaffie JG, Laurienti PJ, Coghill RC (2005) The subjective experience of pain: where expectations become reality. Proc Natl Acad Sci U S A 102:12950-12955.

Lorenz J, Minoshima S, Casey KL (2003) Keeping pain out of mind: the role of the dorsolateral prefrontal cortex in pain modulation. Brain 126:1079-1091.

Mesulam MM, Mufson EJ (1982) Insula of the old world monkey. III: Efferent cortical output and comments on function. J Comp Neurol 212:38-52.

Mufson EJ, Mesulam MM (1982) Insula of the old world monkey. II: Afferent cortical input and comments on the claustrum. J Comp Neurol 212:23-37.

Mufson EJ, Mesulam MM, Pandya DN (1981) Insular interconnections with the amygdala in the rhesus monkey. Neuroscience 6:1231-1248.

Ogawa S, Lee TM, Kay AR, Tank DW (1990) Brain magnetic resonance imaging with contrast dependent on blood oxygenation. Proc Natl Acad Sci U S A 87:9868-9872.

Ohara PT, Vit JP, Jasmin L (2005) Cortical modulation of pain. Cell Mol Life Sci 62:44-52.

Oshiro Y, Quevedo AS, McHaffie JG, Kraft RA, Coghill RC (2007) Brain mechanisms supporting spatial discrimination of pain. J Neurosci 27:3388-3394.

Pedersen JL, Kehlet H (1998a) Hyperalgesia in a human model of acute inflammatory pain: a methodological study. Pain 74:139-151.
Pedersen JL, Kehlet H (1998b) Secondary hyperalgesia to heat stimuli after burn injury in man. Pain 76:377-384.

Petrovic P, Kalso E, Petersson KM, Ingvar M (2002) Placebo and opioid analgesia-imaging a shared neuronal network. Science 295:1737-1740.

Peyron R, Laurent B, García-Larrea L (2000) Functional imaging of brain responses to pain. A review and meta-analysis. Neurophysiol Clin 30:263-288.

Price DD, McHaffie JG, Larson MA (1989) Spatial summation of heatinduced pain: influence of stimulus area and spatial separation of stimuli on perceived pain sensation intensity and unpleasantness. J Neurophysiol 62:1270-1279.

Price DD, Bush FM, Long S, Harkins SW (1994) A comparison of pain measurement characteristics of mechanical visual analogue and simple numerical rating scales. Pain 56:217-226.

Sridharan D, Levitin DJ, Menon V (2008) A critical role for the right frontoinsular cortex in switching between central-executive and default-mode networks. Proc Natl Acad Sci U S A 105:12569-12574.

Tailarach J, Tournoux P (1988) Co-planar stereotaxic atlast of the human brain. New York: Thieme Medical Publishers.

Tölle TR, Kaufmann T, Siessmeier T, Lautenbacher S, Berthele A, Munz F, Zieglgänsberger W, Willoch F, Schwaiger M, Conrad B, Bartenstein P (1999) Region-specific encoding of sensory and affective components of pain in the human brain: a positron emission tomography correlation analysis. Ann Neurol 45:40-47.

Tracey I, Mantyh PW (2007) The cerebral signature for pain perception and its modulation. Neuron 55:377-391.

Woolrich MW, Ripley BD, Brady M, Smith SM (2001) Temporal autocorrelation in univariate linear modeling of FMRI data. Neuroimage $14: 1370-1386$.

Worsley KJ, Evans AC, Marrett S, Neelin P (1992) A three-dimensional statistical analysis for CBF activation studies in human brain. J Cereb Blood Flow Metab 12:900-918.

Zubieta JK, Bueller JA, Jackson LR, Scott DJ, Xu Y, Koeppe RA, Nichols TE, Stohler CS (2005) Placebo effects mediated by endogenous opioid activity on $\mu$-opioid receptors. J Neurosci 25:7754-7762. 